\title{
Lycophyta e samambaias do Parque Nacional da Serra do Itajaí, Vale do Itajaí, SC, Brasil
}

\author{
André Luís de Gasper ${ }^{1,2}$ e Lucia Sevegnani ${ }^{1}$
}

Recebido: 17.09.2009; aceito: 11.11.2010

\begin{abstract}
Lycophyta and ferns from Serra do Itajaí National Park, Vale do Itajaí, SC, Brazil). In this paper we present a floristic survey of the Lycophyta and ferns from the Serra do Itajaí National Park, Santa Catarina. The National Park has an area of 57,475 ha, altitudes varying from 150 to $940 \mathrm{~m}$ and is covered by Atlantic Forest. We have found 190 intraspecific taxa (185 species, three subspecies and two varieties), distributed in 24 families and 73 genera. The most representative genera were: Asplenium (17 species), Thelypteris (10), Blechnum (8), and Lindsaea (7), besides the richest families Dryopteridaceae and Polypodiaceae with 23 and 29 species respectively. Special mention is made to Terpsichore chrysleri (Proctor ex Copel.) A.R. Sm and Polyphlebium hymenophylloides (Bosch) Ebihara \& Dubuisson, which are first records for Santa Catarina.
\end{abstract}

Keys words: Atlantic Forest floristic, South Brazil

RESUMO - (Lycophyta e samambaias do Parque Nacional da Serra do Itajaí, Vale do Itajaí, SC, Brasil). Este trabalho apresenta o levantamento das espécies de Lycophyta e samambaias do Parque Nacional da Serra do Itajaí, Santa Catarina. O Parque possui, no total, 57.475 ha, com altitudes que variam de 150 a 940 m, sendo coberto por Floresta Ombrófila Densa. Foram identificados 190 táxons infragenéricos (185 espécies, três subespécies e duas variedades), pertencentes a 24 famílias e 73 gêneros. Os gêneros mais representativos foram: Asplenium (17 espécies), Thelypteris (10), Blechnum (8) e Lindsaea (7), além da grande riqueza de espécies de Dryopteridaceae e Polypodiaceae, com 23 e 29 espécies, respectivamente. Menção especial deve ser feita para Terpsichore chrysleri (Proctor ex Copel.) A.R. Sm. e Polyphlebium hymenophylloides (Bosch) Ebihara \& Dubuisson que representam em novas referências para Santa Catarina.

Palavras-chave: florística Mata Atlântica, Sul do Brasil

\section{Introdução}

Segundo Morellato \& Haddad (2000), o Bioma Mata Atlântica compreende um conjunto de regiões fitoecológicas, especialmente: Floresta Ombrófila Densa, Floresta Ombrófila Mista, Floresta Ombrófila Aberta, Floresta Estacional Semidecidual, Floresta Estacional Decidual e as formações associadas como pioneira de influência flúvio-marinha (manguezais) e de influência marinha (restingas), todas ricas em biodiversidade. Contudo, este bioma é um dos mais ameaçados pelos constantes desmatamentos e seus remanescentes encontram-se representados em boa parte por florestas secundárias (Mynssen \& Windisch 2004), não restando mais que $11,73 \%$ da floresta original intensamente fragmentada (Ribeiro et al. 2009).
Muito bem representadas no Bioma Mata Atlântica as plantas aqui tratadas como Lycophyta e samambaias, consistem em dois grandes grupos bem distintos em sua origem e evolução (Pryer et al. 2001, 2004, Schneider et al. 2004, Smith et al. 2006). Ocorrem desde regiões semi-desérticas até formações paludosas litorâneas (Page 1979a, b) e têm no Bioma Mata Atlântica um dos três principais centros de endemismo e especiação na América Tropical, que juntamente com o México e a região andina correspondem a $90 \%$ das espécies neotropicais (Tryon 1972).

Estima-se que existam cerca de 13.600 espécies de Lycophyta e samambaias no mundo (Moran 2008), das quais 1.200 a 1.400 ocorrem no Brasil (Prado 1998, Lewinsohn \& Prado 2005). Na região Sul do Brasil as expedições de Roberto Miguel Klein, Padre

1. Universidade Regional de Blumenau, Departamento de Ciências Naturais, Laboratório de Botânica e Herbário FURB, Rua Antônio da Veiga, 140, Bairro Victor Konder, 89012-900 Blumenau, SC, Brasil

2. Autor para correspondência: algasper@gmail.com 
Raulino Reitz, Lyman Smith e do Padre Aloysio Sehnem, no âmbito dos estudos que resultaram na Flora Ilustrada Catarinense (Sehnem 1967a,b,c, 1968a, b, 1970a, b, 1971, 1972, 1974, 1978, 1979a, b, c, d, e, f, g, 1984), catalogaram 420 espécies e, mais recentemente, na publicação da Flora do Cone Sul, Zuloaga et al. (2008) registraram 400 espécies para Santa Catarina.

Especificamente para o vale do Itajaí, uma bacia hidrográfica com $15.500 \mathrm{~km}^{2}$, Klein (1979) apresentou uma lista contendo 206 espécies pertencentes às Lycophyta e samambaias.

O processo histórico e atual de uso do solo, bem como a recente e restrita recuperação da cobertura florestal, resultou em um complexo mosaico vegetacional, composto por estádios sucessionais, áreas com florestas bem conservadas, agricultura e pastagens (Vibrans 2003) tornando difícil afirmar se todas as espécies listadas por Klein (1979) para o Vale do Itajaí ainda ocorrem na região.

$\mathrm{O}$ presente trabalho objetivou realizar o levantamento das espécies de Lycophyta e samambaias, bem como caracterizar os hábitos e ambientes preferenciais destas no Parque Nacional da Serra do Itajaí, SC.

\section{Material e métodos}

O Parque Nacional da Serra do Itajaí (PARNASI) foi criado pelo Decreto Presidencial de 04 de junho de 2004, com 57.475 ha (figura 1) tendo sua

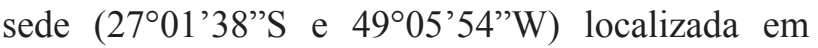
Blumenau, e abrange ainda outros oito municípios de Santa Catarina. A vegetação que o cobre pertence ao bioma Mata Atlântica, região fitoecológica da Floresta Ombrófila Densa, formações Submontana, Montana e Altomontana, segundo o Plano de Manejo do Parque (Gruener 2008). Esta vegetação foi submetida em grande parte a intenso processo histórico de exploração madeireira resultando em um mosaico de floresta primária e floresta secundária avançada. Em menos de 10\% da área do parque houve corte raso da floresta com fins agrícolas ou pecuários.

O clima de região é do tipo $\mathrm{Cfa}$ - temperadoúmido de verão quente, segundo o sistema de classificação de Köppen (1948) com temperatura média anual de $20,1^{\circ} \mathrm{C}$ e médias de inverno não inferiores a $15^{\circ} \mathrm{C}$. A precipitação total anual média é de $1.460 \mathrm{~mm}$, com período mais chuvoso na primavera e verão e a umidade relativa do ar média é elevada, com valores entre $84 \%$ e $86 \%$ (Gaplan 1986).
Os dados foram obtidos em campo no interior do PARNASI ao longo de mais de $50 \mathrm{~km}$ de trilhas e estradas existentes nas localidades denominadas: Sede (Segunda e Terceira Vargem), Subsede (Mono), Faxinal do Bepe, morro do Spitzkopf e Reserva Particular do Patrimônio Natural (RPPN) Bugerkopf, em Blumenau, Indaial e Apiúna, em altitudes entre 150 e $940 \mathrm{~m}$. Foram também analisadas exsicatas coletadas no âmbito do parque, depositados no herbário FURB, da Universidade Regional de Blumenau, Blumenau, SC.

Expedições de três dias de duração foram realizadas mensalmente, por oito meses (março até outubro de 2007). Foram feitas coletas às margens de rios e riachos, barrancos e encostas, afloramentos rochosos, troncos de árvores caídas, interior e borda dos fragmentos e ao longo de trilhas e estradas. Todos os indivíduos férteis foram coletados e incorporados à coleção do herbário FURB. Duplicatas foram enviadas ao Museu Botânico Municipal de Curitiba (MBM), ao Herbário do Departamento de Botânica da Universidade Federal de Minas Gerais (BHCB) e ao Herbário da Universidade Federal do Paraná (UPCB). A coleta e a herborização seguiram metodologia de Fidalgo \& Bononi (1989).

A identificação do material e as informações sobre distribuição geográfica foram obtidas de bibliografia especializada e consulta a especialistas.

As espécies foram classificadas, conforme o hábito, em arborescentes ou herbáceas. As herbáceas foram segregadas em erva terrícola, aquática, rupícola, escandente ou holoepífita (separando-as em holoepífita de samambaias arborescentes ou não). Os ambientes preferenciais foram adaptados de SilvaXavier \& Barros (2005) e podem ser: interior da floresta, borda da floresta, clareira, margem de rio, local aberto (pastagem), encosta, barranco, banhado, margem de córrego ou afloramento, ou paredão rochoso.

A classificação adotada para as samambaias foi a de Smith et al. (2006), os quais reconhecem quatro classes (Psilotopsida, Equisetopsida, Marattiopsida e Polypodiopsida), compreendendo 11 ordens e 37 famílias. Para as Lycophyta, devido à inexistência de classificações recentes, adotou-se a circunscrição de gêneros e famílias adotada por Moran \& Riba (1995) apresentada na Flora Mesoamericana. As abreviações dos nomes dos autores de táxons seguiram PichiSermolli (1996). 


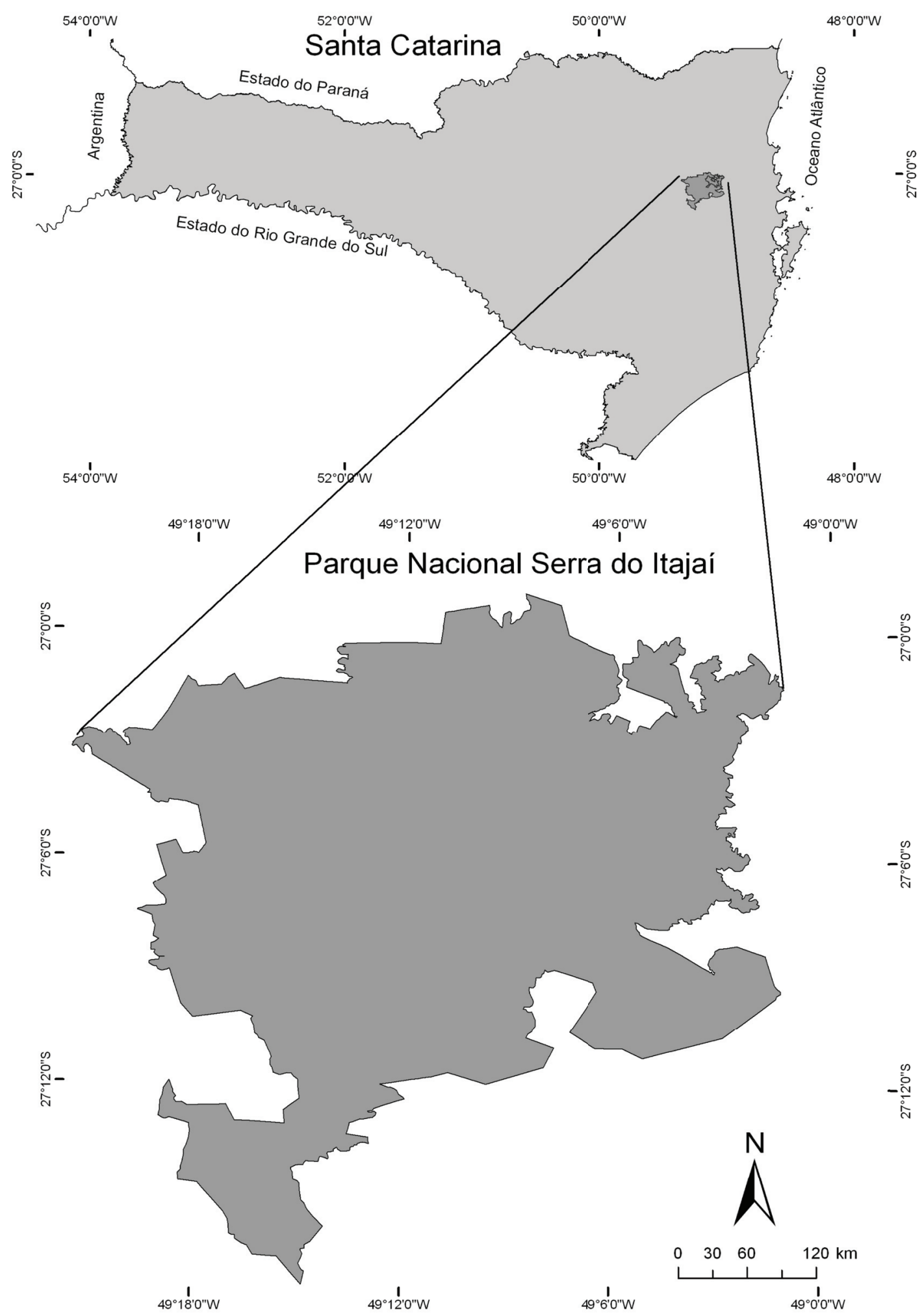

Figura 1. Localização do Parque Nacional da Serra do Itajaí, em Santa Catarina, Brasil.

Figure 1. National Park of Serra do Itajaí, Santa Catarina State, Brazil. 


\section{Resultados e Discussão}

O presente trabalho registrou a ocorrência de 190 táxons infragenéricos (185 espécies, três subespécies e duas variedades) para o Parque Nacional da Serra do Itajaí, distribuídas em 73 gêneros e 24 famílias. Os gêneros mais representativos foram: Asplenium, com 17 espécies, Thelypteris, com 10, Blechnum com oito, e Lindsaea com sete (tabela 1). Os 19 gêneros com maior número de espécies representam $60 \%$ do total amostrado e os outros 49 gêneros representaram outros $40 \%$ das espécies, demonstrando que há gêneros muito diversificados em espécies e outros com menor riqueza na amostra.

As famílias mais representativas foram Polypodiaceae e Dryopteridaceae com 29 e 23 espécies, respectivamente (figura 2). Elevada diversidade nessas duas famílias era esperada, tendo em vista que cerca de $65 \%$ de suas espécies ocorrem em ambientes tropicais (Tryon \& Tryon 1982).

Cabe destacar que Klein (1979) listou 206 espécies de Lycophyta e samambaias com ocorrência no Vale do Itajaí, compreendendo desde ecossistemas litorâneos até as encostas da Serra do Mar e Serra Geral. O PARNASI abrange $4 \%$ da área do vale do Itajaí e até o presente momento abriga cerca de $93 \%$ das espécies citadas por Klein (1979), evidenciando a grande importância desta unidade de conservação para a preservação da biodiversidade desses grupos.

Dentre as espécies citadas por Klein pode-se destacar: Acrostichum danaeifolium Langsd. \& Fisch., de ocorrência em restinga, tipologia vegetacional não abrangida pelo PARNASI. Também não foram encontradas Hemidictyum marginatum (L.) C. Presl e Psilotum nudum (L.) P. Beauv., consideradas por Klein (1979) como muito raras no Vale.

Destaca-se a primeira citação de Terpsichore chrysleri (Copel.) A. R. Sm., bem como de Polyphlebium hymenophylloides (Bosch) Ebihara \& Dubuisson, ampliando a área de ocorrência para estas espécies, antes não conhecidas para Santa Catarina. Vale ressaltar que a Terpsichore chrysleri consta da lista das espécies ameaçadas de extinção do Estado de São Paulo (São Paulo 2004) na categoria vulnerável, tendo sido encontrada no presente trabalho, em apenas uma das trilhas do Parque.

Foi registrada também a ocorrência de Discksonia sellowiana Hook., o xaxim-bugio, espécie ameaçada de extinção (MMA 2008), cujas populações foram submetidas a forte exploração comercial histórica (Windisch 2002). Não foram observadas grandes populações de D. sellowiana no interior do PARNASI, haja vista que crescem preferencialmente em lugares pantanosos e encostas do planalto serrano (Sehnem 1978).

Algumas espécies exóticas subespontâneas (naturalizadas) no continente sul-americano Deparia petersenii (Kunze) Kato, Macrothelypteris torresiana (Gaud.) Ching, Nephrolepis cordifolia (L.) C. Presl e Thelypteris dentata (Forssk.) E. P. St. John foram registradas neste estudo e também por Schwartsburd \& Labiak (2007) como ocorrentes no Parque Estadual de Vila Velha, no Paraná. É costume dos moradores da região do PARNASI o cultivo de $N$. cordifolia como planta ornamental, favorecendo sua disseminação como ruderal. Deparia petersenii, $M$. torresiana e $T$. dentata foram encontradas em áreas florestais do Parque, distantes de antigas residências.

O hábito dos 190 táxons encontrados (figura 3) foi predominantemente herbáceo (183 táxons), mas também foram encontradas espécies com hábito arborescente em Cyatheaceae e Dicksonianaceae, com sete táxons, valores estes muito próximos aos observados por Melo \& Salino (2002) em Minas Gerais. Destaca-se, ainda, que $80 \%$ dos táxons (153) possuem um único hábito e $20 \%$ (37), hábitos variados (tabela 1). Asplenium kunzeanum Klotzsch ex Rosenst., Campyloneurum minus Fée e Thelypteris gymnosora Ponce apresentaram a maior variabilidade de hábito herbáceo, podendo ocorrer como epífitas, epífitas de feto arborescente, terrícolas ou rupícolas (tabela 1).

Especificidade quanto ao forófito foi observada em Asplenium pteropus Kaulf., Pecluma truncorum (Lindm.) M. G. Price e Polyphlebium angustatum (Carmich.) Ebihara \& Dubuisson, as quais, na área de estudo, vicejam apenas em cáudices de Cyatheaceae e Dicksoniaceae. Esta especificidade também foi citada por Senna \& Kazmirczak (1997) para Polyphlebium angustatum e também por Schmitt \& Windisch (2005), que comentam que os cáudices de Cyatheaceae servem de microhábitat para muitas espécies.

Em levantamento de epífitas encontradas sobre Cyatheaceae e Dicksoniaceae na Venezuela, Cortez (2001) registrou a ocorrência de 14 espécies, sendo que Polyphlebium angustatum constava naquele levantamento. Foram registradas, ainda, como epífitas em Cyatheaceae e Dicksoniaceae no PARNASI, Asplenium harpeodes Kunze, $A$. kunzeanum, A. mucronatum C. Presl, A. oligophyllum Kaulf., A. pteropus Kaulf., A. raddianum Gaudich., 
Tabela 1. Espécies amostradas no Parque Nacional da Serra do Itajaí, agrupadas por família conforme Smith et al. (2006) . Hábito $(\mathrm{H}): \mathrm{AR}=$ feto arborescente $\mathrm{TR}=$ terrícolas; $\mathrm{RU}=$ rupícola $; \mathrm{HO}=$ holoepífita; $\mathrm{EP}=$ holoepífita de samambaia arborescente; $\mathrm{ES}=$ escandente; $\mathrm{AQ}=$ aquática. Ambientes preferenciais (AP): $\mathrm{IM}=$ interior da mata; $\mathrm{E}=$ encosta; $\mathrm{AR}=$ afloramento e paredão rochoso; $\mathrm{BM}=$ borda da mata; $\mathrm{LA}=$ local aberto; (pastagem); $\mathrm{B}=$ barranco; $\mathrm{MR}=$ margem de rio; $\mathrm{MC}=$ margem de córrego; $\mathrm{C}=$ clareira . $\mathrm{BA}$ = banhado. O padrão de distribuição geográfica (PD) foi classificado como: $\mathrm{EB}=$ Endêmica do sul do Brasil; $\mathrm{ES}=$ endêmica do Sul e Sudeste; IN = introduzida; PA = pantropical; E = endêmica do Brasil; AS = América do Sul; AT = América Tropical.

Table 1. Species sampled in the National Park of Serra do Itajaí, grouped by family according to Smith et al. (2006). Habit (H): AR $=$ tree fern; $\mathrm{TR}=$ terrestrial; $\mathrm{RU}=$ lithophyte, $\mathrm{HO}=$ holoepiphyte, $\mathrm{EP}=$ epiphyte of tree fern; $\mathrm{ES}=$ terrestrial climber; $\mathrm{AQ}=\mathrm{aquatic}$. Environments (AP): IM = interior of forest, $\mathrm{E}=$ slope, $\mathrm{AR}=$ rocky outcrop or cliff; $\mathrm{BM}=$ forest edge, $\mathrm{LA}=$ open field (pasture), $\mathrm{B}$ $=$ talus, $\mathrm{MR}=$ riverbank, $\mathrm{MC}=$ creekbank; $\mathrm{C}=$ gap; $\mathrm{BA}=$ swamp. The pattern of geographic distribution (PD) was classified as: $\mathrm{EB}$ $=$ endemic to southern Brazil, ES = endemic to South and Southeast IN = introduced, PA = pantropical; $\mathrm{E}=$ endemic to Brazil, $\mathrm{AS}=$ South America, AT = Tropical America.

\begin{tabular}{|c|c|c|c|}
\hline Espécie & $\mathbf{H}$ & $\mathbf{A P}$ & PD \\
\hline \multicolumn{4}{|l|}{ Anemiaceae } \\
\hline Anemia phyllitidis (L.) Sw. & $\mathrm{TR} / \mathrm{RU}$ & $\mathrm{IM} / \mathrm{BM} / \mathrm{B} / \mathrm{MR}$ & AT \\
\hline Anemia raddiana Link & TR/RU & $\mathrm{BM} / \mathrm{LA} / \mathrm{MR} / \mathrm{C}$ & ES \\
\hline Anemia tomentosa (Sav.) Sw. & $\mathrm{TR} / \mathrm{RU}$ & $\mathrm{BM} / \mathrm{LA} / \mathrm{MR}$ & AT \\
\hline \multicolumn{4}{|l|}{ Aspleniaceae } \\
\hline Asplenium abscisum Willd. & $\mathrm{TR}$ & $\mathrm{IM} / \mathrm{MC}$ & AT \\
\hline Asplenium auriculatum $\mathrm{Sw}$. & RU & MR & AT \\
\hline Asplenium brasiliense Sw. & RU & IM & AS \\
\hline Asplenium claussenii Hieron. & TR/RU & IM & AT \\
\hline Asplenium feei Kunze ex Fée & $\mathrm{EP}$ & $\mathrm{BM}$ & AT \\
\hline Asplenium gastonis Fée & $\mathrm{HO}$ & IM & $\mathrm{E}$ \\
\hline Asplenium harpeodes Kunze & $\mathrm{EP}$ & $\mathrm{IM}$ & AS \\
\hline Asplenium incurvatum Fée & $\mathrm{EP}$ & $\mathrm{C}$ & AT \\
\hline Asplenium kunzeanum Klotzsch ex Rosenst. & $\mathrm{HO} / \mathrm{TR} / \mathrm{RU} / \mathrm{EP}$ & $\mathrm{IM} / \mathrm{MC}$ & $\mathrm{E}$ \\
\hline Asplenium martianum C. Chr. & TR & IM & $\mathrm{E}$ \\
\hline Asplenium mucronatum C. Presl & $\mathrm{HO} / \mathrm{EP}$ & $\mathrm{IM} / \mathrm{MC}$ & $\mathrm{E}$ \\
\hline Asplenium oligophyllum Kaulf. & $\mathrm{HO}$ & $\mathrm{IM} / \mathrm{C}$ & AS \\
\hline Asplenium pteropus Kaulf. & $\mathrm{EP} / \mathrm{HO}$ & $\mathrm{IM} / \mathrm{MC}$ & AT \\
\hline Asplenium raddianum Gaudich. & $\mathrm{HO}$ & $\mathrm{IM}$ & AS \\
\hline Asplenium scandicinum Kaulf. & $\mathrm{EP} / \mathrm{HO}$ & $\mathrm{IM}$ & AS \\
\hline Asplenium triquetrum N. Murak. \& R.C. Moran & RU & MR & AT \\
\hline Asplenium uniseriale Raddi & $\mathrm{TR}$ & $\mathrm{E}$ & AS \\
\hline \multicolumn{4}{|l|}{ Blechnaceae } \\
\hline Blechnum binervatum subsp. acutum (Desv.) R.M. Tryon \& Stolze & ES & $\mathrm{IM} / \mathrm{BM}$ & AT \\
\hline Blechnum brasiliense Desv. & TR & $\mathrm{BM} / \mathrm{MR} / \mathrm{C}$ & AT \\
\hline Blechnum cordatum (Desv.) Hieron. & $\mathrm{TR}$ & $\mathrm{IM} / \mathrm{B}$ & AS \\
\hline Blechnum divergens (Kunze) Mett. & $\mathrm{TR}$ & $\mathrm{MC}$ & AT \\
\hline Blechnum occidentale L. & TR & $\mathrm{B}$ & AT \\
\hline Blechnum polypodioides Raddi & TR/RU & $\mathrm{BM} / \mathrm{E} / \mathrm{AR} / \mathrm{B} / \mathrm{MR} / \mathrm{C}$ & AT \\
\hline Blechnum sampaioanum Brade & $\mathrm{TR}$ & $\mathrm{B}$ & $\mathrm{E}$ \\
\hline Blechnum schomburgkii (Klotzsch) C. Chr. & $\mathrm{TR}$ & $\mathrm{BM}$ & $\mathrm{E}$ \\
\hline Salpichlaena volubilis (Kaulf.) J. Sm. & ES & $\mathrm{IM} / \mathrm{BM}$ & AT \\
\hline \multicolumn{4}{|l|}{ Cyatheaceae } \\
\hline Alsophila setosa Kaulf. & $\mathrm{AR}$ & $\mathrm{IM} / \mathrm{BM} / \mathrm{E}$ & AS \\
\hline
\end{tabular}




\begin{tabular}{|c|c|c|c|}
\hline Espécie & $\mathbf{H}$ & AP & PD \\
\hline Cyathea atrovirens (Langsd. \& Fisch.) Domin & $\mathrm{AR}$ & $\mathrm{BM} / \mathrm{E}$ & AS \\
\hline Cyathea corcovadensis Domin & AR & $\mathrm{IM} / \mathrm{BM} / \mathrm{C}$ & E \\
\hline Cyathea delgadii Sternb. & AR & $\mathrm{IM} / \mathrm{BM} / \mathrm{E} / \mathrm{C}$ & $\mathrm{E}$ \\
\hline Cyathea hirsuta C. Presl & AR & $\mathrm{BM}$ & AT \\
\hline Cyathea phalerata Mart. & $\mathrm{AR}$ & $\mathrm{IM}$ & $\mathrm{E}$ \\
\hline \multicolumn{4}{|l|}{ Dennstaedtiaceae } \\
\hline Dennstaedtia cicutaria (Sw.) T. Moore & TR & $\mathrm{IM} / \mathrm{BM}$ & AT \\
\hline Dennstaedtia obtusifolia (Willd.) T. Moore & TR & $\mathrm{IM}$ & AS \\
\hline Histiopteris incisa (Thunb.) J. Sm. & TR & $\mathrm{BM}$ & PA \\
\hline Pteridium arachnoideum (Kaulf.) Maxon & TR & LA & AT \\
\hline \multicolumn{4}{|l|}{ Dicksoniaceae } \\
\hline Dicksonia sellowiana Hook. & $\mathrm{AR}$ & $\mathrm{IM} / \mathrm{C} / \mathrm{MC}$ & AT \\
\hline Lophosoria quadripinnata (J.F. Gmel.) C. Chr. & TR & $\mathrm{BM}$ & AT \\
\hline \multicolumn{4}{|l|}{ Dryopteridaceae } \\
\hline Ctenitis anniesii (Rosenst.) Copel. & TR & $\mathrm{IM} / \mathrm{BM}$ & EB \\
\hline Ctenitis falciculata (Raddi) Ching & TR & $\mathrm{IM} / \mathrm{BM}$ & AS \\
\hline Ctenitis pedicellata (Christ) Copel. & TR & $\mathrm{IM} / \mathrm{BM}$ & $\mathrm{E}$ \\
\hline Didymochlaena truncatula (Sw.) J. Sm. & TR & IM & PA \\
\hline Elaphoglossum balansae C. Chr. & $\mathrm{HO}$ & IM & AS \\
\hline Elaphoglossum lingua Brack. & $\mathrm{HO} / \mathrm{TR}$ & $\mathrm{IM} / \mathrm{BM} / \mathrm{LA} / \mathrm{MR}$ & AT \\
\hline Elaphoglossum luridum (Fée) Christ & $\mathrm{HO}$ & $\mathrm{IM} / \mathrm{MR}$ & AS \\
\hline Elaphoglossum ornatum (Fée) Brade. & $\mathrm{HO}$ & $\mathrm{IM} / \mathrm{MR} / \mathrm{C}$ & AS \\
\hline Elaphoglossum vagans (Mett.) Hieron. & $\mathrm{HO}$ & IM/MR & ES \\
\hline Elaphoglossum strictum (Raddi) T. Moore & RU & IM & AT \\
\hline Lastreopsis amplissima (C. Presl) Tindale. & TR & $\mathrm{IM} / \mathrm{BM} / \mathrm{E} / \mathrm{LA}$ & AS \\
\hline Lastreopsis effusa (C. Presl) Tindale. & TR & IM & AT \\
\hline Megalastrum abundans (Rosenst.) A.R. Sm. \& R.C. Moran & TR & IM & AS \\
\hline Megalastrum connexum (Kaulf.) A.R. Sm. \& R.C. Moran & TR & $\mathrm{BM}$ & AS \\
\hline Megalastrum umbrinum (C. Chr.) A.R. Sm. \& R.C. Moran & TR & $\mathrm{BM}$ & AS \\
\hline Mickelia guianensis (Aubl.) R.C. Moran et al. & ES & IM & AS \\
\hline Olfersia cervina ( L.) Kaulf. & RU & IM & AT \\
\hline Polybotrya cylindrica Kaulf. & ES & $\mathrm{IM} / \mathrm{BM} / \mathrm{C}$ & $\mathrm{E}$ \\
\hline Polystichum montevidense (Spreng.) Rosenst. & TR & $\mathrm{IM} / \mathrm{C}$ & AS \\
\hline Rumohra adiantiformis (G. Forst.) Ching & TR & LA & PA \\
\hline Stigmatopteris brevinervis (Fée) R.C. Moran & TR & $\mathrm{BM}$ & $\mathrm{E}$ \\
\hline Stigmatopteris caudata (Raddi) C. Chr. & TR & $\mathrm{IM}$ & $\mathrm{E}$ \\
\hline Stigmatopteris heterocarpa (Fée) Rosenst. & TR & $\mathrm{IM} / \mathrm{BM} / \mathrm{E}$ & $\mathrm{E}$ \\
\hline \multicolumn{4}{|l|}{ Gleicheniaceae } \\
\hline Dicranopteris flexuosa (Schrad.) Underw. & TR & LA & AT \\
\hline Dicranopteris nervosa (Kaulf.) Maxon & TR & LA & AS \\
\hline Gleichenella pectinata (Willd.) Ching & TR & LA & AT \\
\hline Sticherus bifidus (Willd.) Ching & TR & $\mathrm{BM} / \mathrm{LA} / \mathrm{MR} / \mathrm{C}$ & AT \\
\hline Sticherus nigropaleaceus (Sturm) J. Prado & TR & $\mathrm{BM}$ & AS \\
\hline Sticherus pubescens (Humb. \& Bonpl. ex Willd.) Nakai & $\mathrm{TR}$ & LA & AS \\
\hline Sticherus squamosus (Fée) J. Gonzáles & TR & $\mathrm{BM}$ & $\mathrm{E}$ \\
\hline
\end{tabular}

\section{Hymenophyllaceae}




\begin{tabular}{|c|c|c|c|}
\hline Espécie & $\mathbf{H}$ & $\mathbf{A P}$ & PD \\
\hline Abrodictyum rigidum (Sw.) Ebihara \& Dubuisson & TR & IM & $\mathrm{AT}$ \\
\hline Hymenophyllum asplenioides (Sw.) Sw. & $\mathrm{HO}$ & $\mathrm{IM}$ & AS \\
\hline Hymenophyllum caudiculatum Mart. & $\mathrm{HO}$ & $\mathrm{IM}$ & AT \\
\hline Hymenophyllum hirsutum (L.) Sw. & $\mathrm{HO}$ & IM & PA \\
\hline Hymenophyllum polyanthos Sw. & $\mathrm{HO}$ & IM & AT \\
\hline Polyphlebium angustatum (Carmich.) Ebihara \& Dubuisson & EP & $\mathrm{IM}$ & AT \\
\hline Polyphlebium diaphanum (Kunth) Ebihara \& Dubuisson & RU & MR & AT \\
\hline Polyphlebium hymenophylloides (Bosch) Ebihara \& Dubuisson & EP & MR & AT \\
\hline Polyphlebium pyxidiferum (L.) Ebihara \& Dubuisson & EP & $\mathrm{IM}$ & $\mathrm{AT}$ \\
\hline Trichomanes elegans Rich. & TR/RU & $\mathrm{IM} / \mathrm{E}$ & AT \\
\hline Trichomanes emarginatum C. Presl & $\mathrm{EP}$ & IM & AS \\
\hline Trichomanes pilosum Raddi & TR & $\mathrm{B} / \mathrm{C} / \mathrm{E}$ & AS \\
\hline Trichomanes polypodioides L. & $\mathrm{HO} / \mathrm{EP}$ & IM & AT \\
\hline Vandenboschia radicans (Sw) Copel. & ES & IM & PA \\
\hline Vandenboschia rupestris (Raddi) Ebihara \& K. Iwats. & $\mathrm{HO}$ & IM & PA \\
\hline \multicolumn{4}{|l|}{ Lindsaeaceae } \\
\hline Lindsaea arcuata Kunze & TR & IM & $\mathrm{AT}$ \\
\hline Lindsaea bifida (Kaulf.) Mett. ex Kuhn & TR & $\mathrm{IM} / \mathrm{E}$ & $\mathrm{E}$ \\
\hline Lindsaea lancea (L.) Bedd. & $\mathrm{TR} / \mathrm{RU}$ & $\mathrm{IM} / \mathrm{BM} / \mathrm{E}$ & AT \\
\hline Lindsaea ovoidea Fée & $\mathrm{TR} / \mathrm{RU}$ & $\mathrm{MR} / \mathrm{E}$ & $\mathrm{E}$ \\
\hline Lindsaea quadrangularis Raddi subsp pallecens Sehnem & TR/RU & $\mathrm{IM} / \mathrm{BM} / \mathrm{E} / \mathrm{AR}$ & EB \\
\hline Lindsaea quadrangularis Raddi subsp terminalis K.U. Kramer & TR & $\mathrm{IM} / \mathrm{BM} / \mathrm{E} / \mathrm{B} / \mathrm{C}$ & AS \\
\hline Lindsaea quadrangularis Raddi subsp quadrangularis K.U. Kramer & TR & IM & ES \\
\hline \multicolumn{4}{|l|}{ Lomariopsidaceae } \\
\hline Lomariopsis marginata (Schrad.) Kuhn & ES & IM & $\mathrm{E}$ \\
\hline Nephrolepis cordifolia (L.) C. Presl & TR & LA & IN \\
\hline Nephrolepis pendula (Raddi) Fée & $\mathrm{HO}$ & IM & AT \\
\hline \multicolumn{4}{|l|}{ Lycopodiaceae } \\
\hline Huperzia acerosa (Sw.) Holub & $\mathrm{HO}$ & $\mathrm{IM}$ & AT \\
\hline Huperzia comans (Nessel) B. Øllg. \& P.G. Windisch & $\mathrm{HO}$ & IM & ES \\
\hline Huperzia flexibilis (Fée) B. Øllg. & $\mathrm{HO}$ & $\mathrm{IM} / \mathrm{BM}$ & $\mathrm{E}$ \\
\hline Huperzia heterocarpon (Fée) Holub & $\mathrm{HO}$ & $\mathrm{C}$ & AS \\
\hline Huperzia hexasticha B. Øllg. \& P.G. Windisch & $\mathrm{HO}$ & $\mathrm{IM}$ & ES \\
\hline Huperzia mandiocana (Raddi) Trevisan & $\mathrm{HO}$ & IM & AS \\
\hline Huperzia reflexa (Lam.) Trevisan & $\mathrm{TR}$ & B & AT \\
\hline Lycopodiella camporum B. Øllg. \& P.G. Windisch & TR & LA & AT \\
\hline Lycopodiella caroliniana (L.) Pc. Serm. & TR & LA & PA \\
\hline Lycopodiella cernua (L.) Pic. Serm. & TR/RU & $\mathrm{BM} / \mathrm{LA} / \mathrm{B}$ & PA \\
\hline Lycopodium clavatum $\mathrm{L}$. & $\mathrm{TR}$ & BM/LA & PA \\
\hline Lycopodium thyoides Humb. \& Bonpl. ex Willd. & TR & LA & AT \\
\hline \multicolumn{4}{|l|}{ Marattiaceae } \\
\hline Danaea geniculata Raddi & $\mathrm{TR}$ & $\mathrm{IM}$ & AT \\
\hline Danaea moritziana C. Presl & TR & $\mathrm{IM} / \mathrm{E} / \mathrm{B}$ & AT \\
\hline Eupodium kaulfussii (J. Sm.) Hook. & $\mathrm{TR}$ & $\mathrm{AR} / \mathrm{B}$ & $\mathrm{E}$ \\
\hline Marattia cicutifolia Kaulf. & TR & $\mathrm{MC}$ & $\mathrm{AS}$ \\
\hline
\end{tabular}




\section{Espécie}

Ophioglossaceae
Ophioglossum palmatum L.

Osmundaceae

Osmunda regalis $\mathrm{L}$.

Osmundastrum cinnamomeum (L.) C. Presl

Polypodiaceae

Alansmia reclinata (Brack.) Labiak

Campyloneurum acrocarpon Fée

Campyloneurum minus Fée

Campyloneurum nitidum (Kaulf.) C. Pres1

Campyloneurum rigidum J. Sm.

Ceradenia spixiana (Mart. ex Mett.) L.E. Bishop

Cochlidium punctatum (Raddi) L.E. Bishop

Cochlidium serrulatum (Sw.) L.E. Bishop

Lellingeria brevistipes (Mett. ex Kuhn) A.R. Sm. \& R.C. Moran

Leocotrichum schenckii (Hieron.) Labiak

Microgramma percussa (Cav.) de la Sota

Microgramma squamulosa (Kaulf.) de la Sota

Microgramma tecta (Kaulf.) Alston

Microgramma vacciniifolia (Langsd. \& Fisch.) Copel.

Micropolypodium achilleifolium (Kaulf.) Labiak \& F.B. Matos

Niphidium crassifolium (L.) Lellinger

Pecluma chnoophora (Kunze) Salino \& Costa Assis

Pecluma paradiseae (Langsd. \& Fisch.) M.G. Price

Pecluma recurvata (Kaulf.) M.G. Price

Pecluma truncorum (Lindm.) M.G. Price

Pleopeltis astrolepis Liebm.

Pleopeltis hirsutissima (Raddi) de la Sota

Pleopeltis macrocarpa (Bory ex Willd.) Kaulf.

Pleopeltis pleopeltifolia (Raddi) Alston

Serpocaulon catharinae (Langsd. \& Fisch.) A.R. Sm.

Serpocaulon fraxinifolium (Jacq.) A.R. Sm.

Serpocaulon latipes (Langsd. \& Fisch.) A.R. Sm.

Serpocaulon vacillans (Link) A.R. Sm.

Terpsichore chrysleri (Copel.) A.R. Sm.

\section{Pteridaceae}

Adiantopsis chlorophylla (Sw.) Fée

Adiantum curvatum Kaulf.

Adiantum pentadactylon Langsd. \& Fisch.

Adiantum raddianum C. Presl

Doryopteris crenulans (Fée) Christ

Doryopteris pentagona Pic. Serm.

Jamesonia myriophylla (Sw.) Christenh.

Pityrogramma calomelanos (L.) Link

Pteris altissima Poir.

Pteris decurrens C. Presl
H

$\mathrm{HO}$

IM

AP

PD

TR

BM

BA

PA

$\mathrm{HO}$

IM

ES

TR

HO/TR/EP

$\mathrm{HO} / \mathrm{TR}$

$\mathrm{HO}$

$\mathrm{HO}$

$\mathrm{HO}$

$\mathrm{HO} / \mathrm{TR}$

$\mathrm{HO}$

$\mathrm{HO}$

$\mathrm{HO} / \mathrm{RU}$

$\mathrm{HO}$

$\mathrm{HO}$

$\mathrm{HO}$

$\mathrm{HO}$

$\mathrm{HO} / \mathrm{RU}$

$\mathrm{HO} / \mathrm{RU}$

$\mathrm{HO}$

$\mathrm{HO}$

EP

$\mathrm{HO}$

$\mathrm{HO} / \mathrm{RU}$

$\mathrm{HO} / \mathrm{EP}$

$\mathrm{HO}$

$\mathrm{HO}$

$\mathrm{HO}$

TR

TR

$\mathrm{HO}$

TR

TR

TR

TR/RU

TR

TR

TR

TR

TR

TR
IM

$\mathrm{IM} / \mathrm{E} / \mathrm{AR} / \mathrm{C} / \mathrm{MC}$

$\mathrm{IM} / \mathrm{BM} / \mathrm{C}$

IM

$\mathrm{BM} / \mathrm{MC}$

IM

$\mathrm{IM} / \mathrm{MR}$

IM

IM

$\mathrm{BM} / \mathrm{AR} / \mathrm{MR}$

$\mathrm{BM} / \mathrm{C}$

$\mathrm{BM}$

$\mathrm{BM} / \mathrm{LA} / \mathrm{C}$

IM

$\mathrm{BM} / \mathrm{MR}$

$\mathrm{IM} / \mathrm{AR}$

MR

IM/BM/MR

IM

IM/BM

IM/BM/LA

$\mathrm{BM} / \mathrm{LA}$

$\mathrm{BM} / \mathrm{C}$

$\mathrm{BM} / \mathrm{LA}$

IM/BM

LA

IM

IM

E

AS

AS

AS

AT

E

PA

ES

ES

AT

AS

AT

AS

E

AT

AS

E

AS

AS

AT

AS

PA

AS

AS

AT

AT

AS

AS

AS

AT

E

AS

AS

AS

AS

AT

AT

AT 


\begin{tabular}{|c|c|c|c|}
\hline Espécie & $\mathbf{H}$ & AP & PD \\
\hline Pteris lechleri Mett. & TR & $\mathrm{IM}$ & AT \\
\hline Pteris podophylla $\mathrm{Sw}$. & TR & $\mathrm{MC}$ & $\mathrm{E}$ \\
\hline Pteris splendens Kaulf. & TR & $\mathrm{IM}$ & AS \\
\hline Radiovittaria stipitata (Kunze) E.H. Crane & $\mathrm{HO}$ & IM & AT \\
\hline Vittaria lineata (L.) Sm. & $\mathrm{HO}$ & $\mathrm{IM} / \mathrm{MR} / \mathrm{C}$ & AT \\
\hline Vittaria scabrida Klotzsch ex Fée & $\mathrm{HO}$ & $\mathrm{IM} / \mathrm{MR}$ & AS \\
\hline \multicolumn{4}{|l|}{ Saccolomataceae } \\
\hline Saccoloma elegans Kaulf. & $\mathrm{TR}$ & $\mathrm{IM}$ & AT \\
\hline Saccoloma inaequale (Kunze) Mett. & TR & $\mathrm{IM} / \mathrm{E}$ & AT \\
\hline \multicolumn{4}{|l|}{ Salviniaceae } \\
\hline Salvinia minima Baker & AQ & LA & AT \\
\hline \multicolumn{4}{|l|}{ Schizaeaceae } \\
\hline Lygodium volubile $\mathrm{Sw}$. & ES & $\mathrm{BM}$ & PA \\
\hline Schizaea elegans (Vahl) Sw. & $\mathrm{TR}$ & $\mathrm{E}$ & AT \\
\hline \multicolumn{4}{|l|}{ Selaginellaceae } \\
\hline Selaginella contigua Baker & $\mathrm{TR} / \mathrm{RU}$ & $\mathrm{IM} / \mathrm{BM} / \mathrm{B} / \mathrm{MC}$ & $\mathrm{E}$ \\
\hline Selaginella decomposita Spring & $\mathrm{TR}$ & $\mathrm{C}$ & $\mathrm{E}$ \\
\hline Selaginella flexuosa Spring & $\mathrm{TR} / \mathrm{RU}$ & $\mathrm{IM} / \mathrm{BM} / \mathrm{B} / \mathrm{C} / \mathrm{MC}$ & AS \\
\hline Selaginella muscosa Spring & RU & BM & AS \\
\hline Selaginella sulcata (Desv. ex Poir.) Spring ex Mart. & RU & MR & AS \\
\hline \multicolumn{4}{|l|}{ Tectariaceae } \\
\hline Tectaria buchtienii (Rosenst.) Maxon & TR & $\mathrm{IM}$ & EB \\
\hline Tectaria incisa Cav. & TR & $\mathrm{IM} / \mathrm{C}$ & AT \\
\hline Tectaria pilosa (Fée) R.C. Moran & TR & $\mathrm{IM} / \mathrm{MR}$ & AT \\
\hline \multicolumn{4}{|l|}{ Thelypteridaceae } \\
\hline Macrothelypteris torresiana (Gaud.) Ching & TR & $\mathrm{C}$ & IN \\
\hline Thelypteris amambayensis (Christ) Ponce & $\mathrm{TR}$ & $\mathrm{IM} / \mathrm{BM} / \mathrm{C}$ & AS \\
\hline Thelypteris dentata (Forssk.) E. St. John & $\mathrm{TR} / \mathrm{RU}$ & $\mathrm{BM}$ & IN \\
\hline Thelypteris gymnosora Ponce & $\mathrm{HO} / \mathrm{TR}$ & $\mathrm{BM} / \mathrm{MR} / \mathrm{MC}$ & AT \\
\hline Thelypteris opposita (Vahl) Ching & TR & $\mathrm{BM}$ & $\mathrm{E}$ \\
\hline Thelypteris pachyrhachis (Kunze ex Mett.) Ching var. pachyrhachis & TR & B & $\mathrm{E}$ \\
\hline Thelypteris ptarmica (Kunze) C.F. Reed & $\mathrm{TR} / \mathrm{RU}$ & MR & AT \\
\hline Thelypteris raddii (Rosenst.) Ponce & $\mathrm{TR} / \mathrm{RU}$ & $\mathrm{IM} / \mathrm{MR}$ & $\mathrm{E}$ \\
\hline Thelypteris retusa (Sw.) C.F. Reed & TR & $\mathrm{BM}$ & E \\
\hline Thelypteris serrata (Cav.) Alston & $\mathrm{TR}$ & $\mathrm{IM}$ & AT \\
\hline Thelypteris vivipara (Raddi) C.F. Reed & $\mathrm{TR} / \mathrm{RU}$ & $\mathrm{IM}$ & $\mathrm{E}$ \\
\hline \multicolumn{4}{|l|}{ Woodsiaceae } \\
\hline Deparia petersenii (Kunze) Kato & TR & $\mathrm{BM} / \mathrm{B} / \mathrm{MR}$ & IN \\
\hline Diplazium ambiguum Raddi & TR & $\mathrm{BM}$ & AT \\
\hline Diplazium cristatum (Desr.) Alston & $\mathrm{TR} / \mathrm{RU}$ & $\mathrm{BM} / \mathrm{E} / \mathrm{B} / \mathrm{C}$ & AT \\
\hline Diplazium lindbergii (Mett.) Christ & $\mathrm{TR}$ & $\mathrm{BM}$ & AT \\
\hline Diplazium plantaginifolium (L.) Urb. & TR & $\mathrm{IM}$ & AS \\
\hline Diplazium riedelianum (Bong. ex Kuhn) Kuhn ex C. Chr. & TR & $\mathrm{IM}$ & AT \\
\hline
\end{tabular}




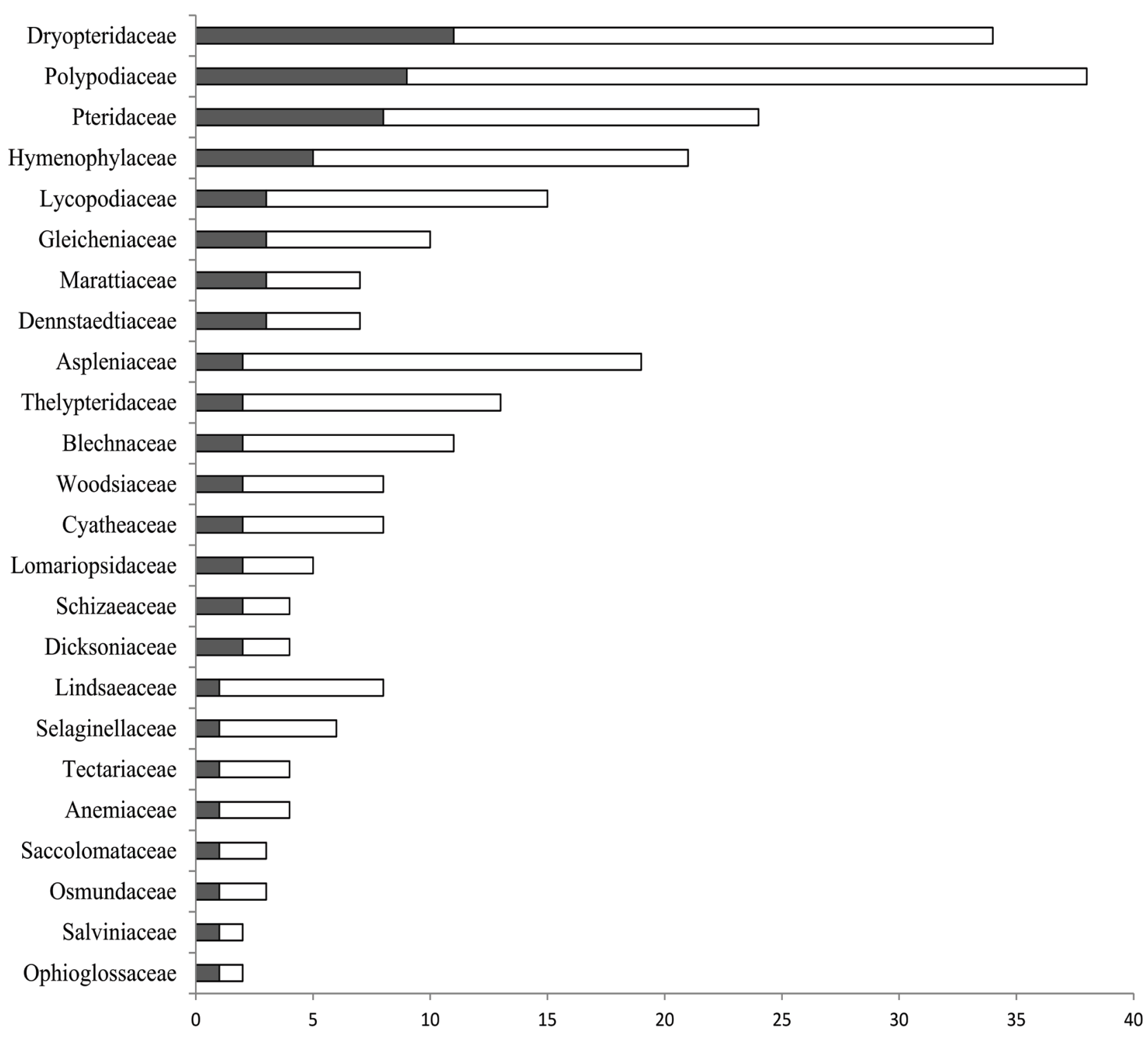

Figura 2. Relação gênero (ロ) e espécie ( $\square$ ) das Lycophyta e samambaias encontradas no Parque Nacional da Serra do Itajaí, Santa Catarina, Brasil.

Figure 2. Genera (घ) and species ( $\square$ ) of Lycophyta and Ferns from National Park of Serra do Itajaí, Santa Catarina, Brazil. 


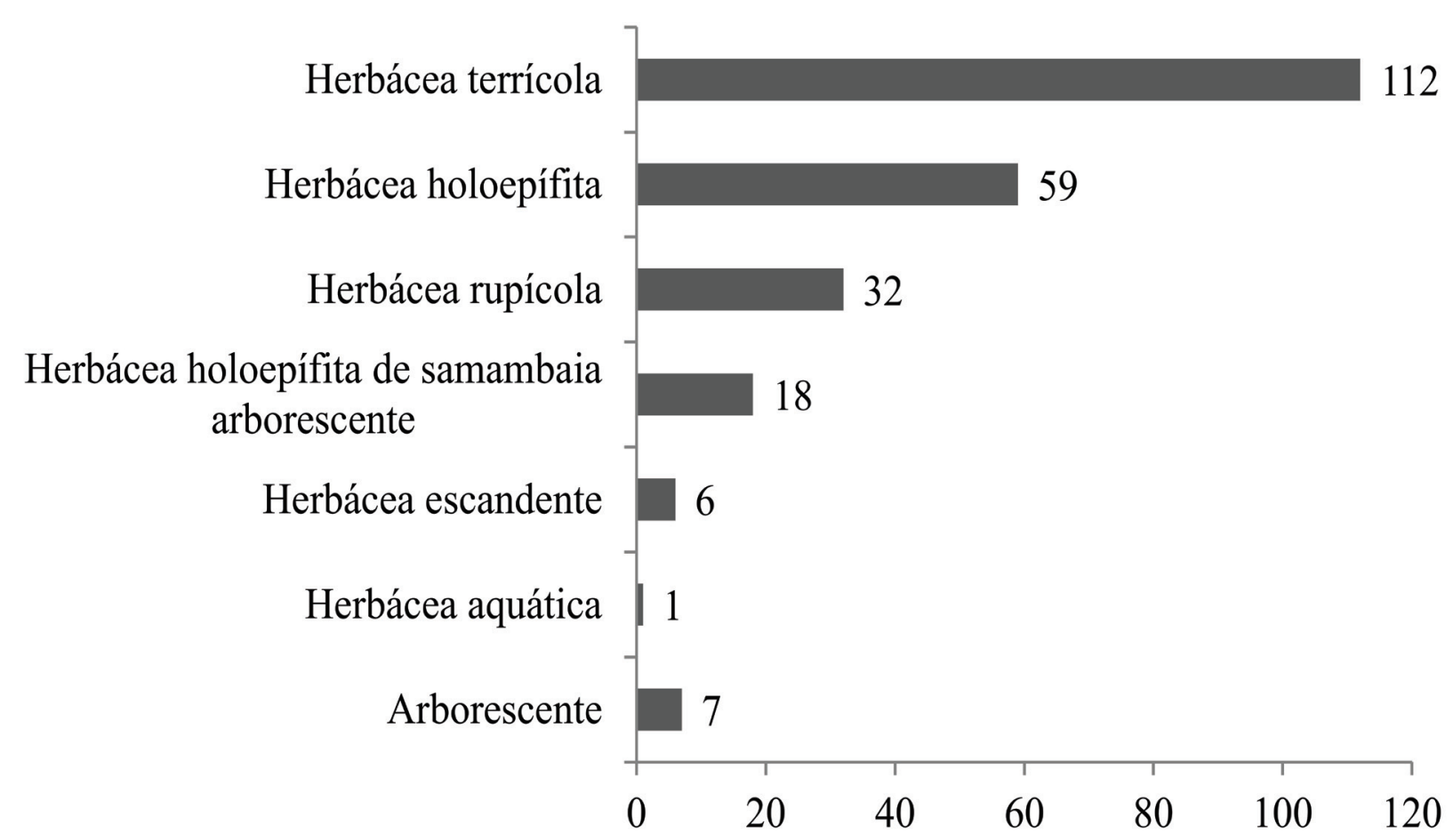

Figura 3. Hábito das espécies de Lycophyta e Samambaia do Parque Nacional da Serra do Itajaí. Figure 3. Habit of the species of Lycophta and Ferns of National Park of Serra do Itajaí.

A. scandicinum Kaulf., Campyloneurum minus, Pecluma truncorum, Pleopeltis macrocarpa (Bory ex Willd.) Kaulf., P. pleopeltifolia Raddi Alston, Selaginella flexuosa Spring, Terpsichore chrysleri, Thelypteris gymnosora, Trichomanes polypodioides L. e Vandenboschia radicans (Sw.) Copel. totalizando, 18 espécies.

Em relação aos ambientes ocupados na área de estudo: 34,6\% (108 táxons) foram observados no interior da floresta, $21,1 \%$ (67) na borda da floresta, $8,8 \%$ (28) em clareira, $8,8 \%$ (28) na margem de rio, $8,5 \%$ (27) em local aberto (pastagem), 6,3\% (20) em encosta, 5,3\% (17) em barranco, 4,4\% (14) em margem de córrego, $1,9 \%$ (6) em afloramento e paredão rochoso e apenas $0,3 \%$ (1) em banhado.

Ocorrendo em apenas um ambiente preferencial foram encontradas 118 espécies, enquanto Blechnum polypodioides Raddi, Campyloneurum minus, Lindsaea quadrangularis Raddi subsp. terminalis K.U. Kramer e Selaginella flexuosa foram observadas ocorrendo em cinco ou mais ambientes, demonstrando que essas espécies são generalistas na área de estudo.

Quanto à distribuição geográfica das espécies, 8\% (15 táxons) são de ocorrência pantropical e $2 \%$ (4) são introduzidos (tabela 1). Dentre os de ocorrência nas Américas: 38\% (72) são de ocorrência na América Tropical, 29\% (55) na América do Sul, $17 \%$ (33) são endêmicas do Brasil, sendo ainda 4\% (8) endêmicas das regiões Sul e Sudeste do Brasil e $2 \%$ (3), endêmicas apenas da região Sul do Brasil.

A grande diversidade de espécies e seus hábitos registrada por este trabalho evidencia a heterogeneidade ambiental e o bom estado de conservação do Parque Nacional da Serra do Itajaí, destacando-o como importante remanescente florestal do bioma Mata Atlântica e relevante abrigo para a biota autóctone. 


\section{Agradecimentos}

Os autores agradecem em especial aos Drs. Alexandre Salino, Vinícius A. O. Dittrich, Paulo H. Labiak e Jefferson Prado pela ajuda na identificação e envio de material e, especialmente, a M.Sc. Rosane Hiendlmayer pelo auxilio nas primeiras identificações. Agradecemos ainda as prestimosas contribuições dos revisores e editores. Ao ICMBio pela autorização de coleta, ao Herbário FURB e ao Laboratório de Botânica da Universidade Regional de Blumenau pelo auxílio no processamento do material, e ao Departamento de Ciências Naturais por possibilitar o deslocamento ao campo.

\section{Literatura citada}

Cortez, L. 2001. Pteridofitas epifitas encontradas en Cyatheaceae y Dicksoniaceae de los bosques nublados de Venezuela. Gayana Botánica 58: 13-23.

Fidalgo, O. \& Bononi, V.L.R. 1989. Técnicas de coleta, preservação e herborização de material botânico. Instituto de Botânica, São Paulo.

Gaplan. 1986. Atlas de Santa Catarina. Aerofoto Cruzeiro, Rio de Janeiro.

Gruener, C.G. 2008. Plano de manejo Parque Nacional da Serra do Itajaí. Acaprena, Blumenau.

Klein, R.M. 1979. Ecologia da flora e vegetação do Vale do Itajaí. Sellowia 31: 1-165.

Köppen, W. 1948. Climatologia; con un estudio de los climas de la tierra. Fondo de Cultura Economica, México.

Labiak, P.H. \& Prado, J. 2005. As espécies de Terpsichore A.R. Sm. e Zygophlebia L.E. Bishop (Grammitidaceae) do Brasil. Acta Botanica Brasilica 19: 867-887.

Lewinsohn, T.M. \& Prado, P.I. 2005. Quantas espécies há no Brasil? Megadiversidade 1: 36-42.

Melo, L.C.N. \& Salino, A. 2002. Pteridófitas de duas áreas de floresta da Bacia do Rio Doce no Estado de Minas Gerais, Brasil. Lundiana 3: 129-139.

MMA (Ministério do Meio Ambiente). 2008. Lista oficial de espécies da flora brasileira ameaçada de extinção. Diário Oficial da União de 24 de setembro de 2008. no 185. Seção 1, pp.75-83.

Moran, R.C. \& Riba, R. (eds.). 1995. Psilotaceae a Salviniaceae. In: G. Davidse, M. Souza \& S. Knapp (eds.). Flora Mesoamericana. Universidad Nacional Autónoma de México, México, v.1, pp. 1-470.

Moran, R.C. 2008. Diversity, biogeography, and floristics. In: T.A. Ranker \& C.H. Haufler (eds.). Biology and evolution of ferns and lycophytes. Cambridge University Press, Cambridge, pp. 367-394.

Morellato, L.P. \& Haddad, C.F.B. 2000. Introduction: the brazilian atlantic forest. Biotropica 32: 786-792.

Mynssen, C.M. \& Windisch, P.G. 2004. Pteridófitas da Reserva Rio das Pedras, Mangaratiba, RJ, Brasil. Rodriguésia 55: 125-156.

Page, C.N. 1979a. The diversity of ferns: an ecological perspective. In: A.F. Dyer (ed.). The experimental biology of ferns. Academic Press, London, pp. 10-56.

Page, C.N. 1979b. Experimental aspects of fern ecology. In: A.F. Dyer (ed.). The experimental biology of ferns. Academic Press, London, pp. 551-589.

Prado, J. 1998. Pteridófitas do Estado de São Paulo. In: C.E.M. Bicudo \& G.J. Shepherd (eds.). Biodiversidade do Estado de São Paulo: Síntese do conhecimento ao final do século XX Fungos macroscópicos e plantas. FAPESP, São Paulo, pp. 49-61.

Pichi-Sermolli, R.E.G. 1996. Authors of scientific names in Pteridophyta. Royal Botanic Gardens, Kew.

Pryer, K.M., Schneider, H., Smith, A.R., Cranfill, R., Wolf, P.G., Hunt, J.S. \& Sipes, S.D. 2001. Horsetails and ferns are a monophyletic group and the closest living relatives to seed plants. Nature 409: 618-622.

Pryer, K.M., Schneider, H. \& Magallón, S. 2004. The radiation of vascular plants. In: J. Cracraft \& M.J. Donoghue (eds.). Assembling the Tree of Life. Oxford University Press, London, pp. 138-153.

Ribeiro, M.C., Metzger J.P., Martensen, A.C., Ponzoni F.J. \& Hirota M.M. 2009. The Brazilian Atlantic Forest: How much is left, and how is the remaining forest distributed? Implications for conservation. Biological Conservation 142: 1141-53.

São Paulo. 2004. Lista das espécies da flora ameaçadas de extinção. Resolução SMA-48, de 21.09.2004, D.O.E. 22/09/2004, Seção I, pp. 1-3.

Schmitt, J.L. \& Windisch, P.G. 2005. Aspectos ecológicos de Alsophila setosa Kaulf. (Cyatheaceae, Pteridophyta) no Rio Grande do Sul, Brasil. Acta Botanica Brasilica 19: 859-865.

Schneider, H., Schuettpelz, E., Pryer, K.M., Cranfill, R., Magallón, S. \& Lupia, R. 2004. 
Ferns diversified in the shadow of angiosperms. Nature 428: 553-557.

Schwartsburd, P.B. \& Labiak, P.H. 2007. Pteridófitas do Parque Estadual de Vila Velha, Ponta Grossa, Paraná, Brasil. Hoehnea 34: 159-209.

Sehnem, A. 1967a. Maratiáceas. In: R. Reitz (ed.). Flora Ilustrada Catarinense. Herbário Barbosa Rodrigues, Itajaí.

Sehnem, A. 1967b. Osmundáceas. In: R. Reitz (ed.). Flora Ilustrada Catarinense. Herbário Barbosa Rodrigues, Itajaí.

Sehnem, A. 1967c. Plagiogiriáceas. In: R. Reitz (ed.). Flora Ilustrada Catarinense. Herbário Barbosa Rodrigues, Itajaí.

Sehnem, A. 1967d. Vitariáceas. In: R. Reitz (ed.). Flora Ilustrada Catarinense. Herbário Barbosa Rodrigues, Itajaí.

Sehnem, A. 1968a. Aspleniáceas. In: R. Reitz (ed.). Flora Ilustrada Catarinense. Herbário Barbosa Rodrigues, Itajaí.

Sehnem, A. 1968b. Blecnáceas. In: R. Reitz (ed.). Flora Ilustrada Catarinense. Herbário Barbosa Rodrigues, Itajaí.

Sehnem, A. 1970a. Gleiqueniáceas. In: R. Reitz (ed.). Flora Ilustrada Catarinense. Herbário Barbosa Rodrigues, Itajaí.

Sehnem, A. 1970b. Polipodiáceas. In: R. Reitz (ed.). Flora Ilustrada Catarinense. Herbário Barbosa Rodrigues, Itajaí.

Sehnem, A. 1971. Himenofiláceas. In: R. Reitz (ed.). Flora Ilustrada Catarinense. Herbário Barbosa Rodrigues, Itajaí.

Sehnem, A. 1972. Pteridáceas. In: R. Reitz (ed.). Flora Ilustrada Catarinense. Herbário Barbosa Rodrigues, Itajaí.

Sehnem, A. 1974. Esquizeáceas. In: R. Reitz (ed.). Flora Ilustrada Catarinense. Herbário Barbosa Rodrigues, Itajaí.

Sehnem, A. 1978. Ciateáceas. In: R. Reitz (ed.). Flora Ilustrada Catarinense. Herbário Barbosa Rodrigues, Itajaí.

Sehnem, A. 1979a. Aspidiáceas. In: R. Reitz (ed.). Flora Ilustrada Catarinense. Herbário Barbosa Rodrigues, Itajaí.

Sehnem, A. 1979b. Davaliáceas. In: R. Reitz (ed.). Flora Ilustrada Catarinense. Herbário Barbosa Rodrigues, Itajaí.

Sehnem, A. 1979c. Marsiliáceas. In: R. Reitz (ed.). Flora Ilustrada Catarinense. Herbário Barbosa Rodrigues, Itajaí.

Sehnem, A. 1979d. Ofioglossáceas. In: R. Reitz (ed.).
Flora Ilustrada Catarinense. Herbário Barbosa Rodrigues, Itajaí.

Sehnem, A. 1979e. Parkeriáceas. In: R. Reitz (ed.). Flora Ilustrada Catarinense. Herbário Barbosa Rodrigues, Itajaí.

Sehnem, A. 1979f. Psilotáceas. In: R. Reitz (ed.). Flora Ilustrada Catarinense. Herbário Barbosa Rodrigues, Itajaí.

Sehnem, A. 1979g. Salviniáceas. In: R. Reitz (ed.). Flora Ilustrada Catarinense. Herbário Barbosa Rodrigues, Itajaí.

Sehnem, A. 1983. Chave para as famílias de pteridófitos da Região Sul do Brasil. In: R. Reitz (ed.). Flora Ilustrada Catarinense. Herbário Barbosa Rodrigues, Itajaí.

Sehnem, A. 1984. Equisetáceas. In: R. Reitz (ed.). Flora Ilustrada Catarinense. Herbário Barbosa Rodrigues, Itajaí.

Senna, R.M. \& Kazmirczak, C. 1997. Pteridófitas de um remanescente florestal no Morro da Extrema, Porto Alegre, RS. Faculdade de Zootecnia, Veterinária e Agronomia 4: 47-57.

Silva-Xavier, S.R.S. \& Barros, I.C.L. 2005. Pteridoflora e seus aspectos ecológicos ocorrentes no Parque Ecológico João Vasconcelos Sobrinho, Caruaru, PE, Brasil. Acta Botanica Brasilica 19: 777-781.

Smith, A., Pryer, K., Schuettpelz, E., Korall, P., Schneider, H. \& Wolf, P. 2006. A classification for extant ferns. Taxon 55: 705-731.

Tryon, R.M. \& Tryon, A.F. 1982. Ferns and allied plants with special reference to tropical America. Springer - Verlag, New York.

Tryon, R.M. 1972. Endemic areas and geographical speciation in tropical american ferns. Biotropica 4: $121-131$.

Vibrans, A.C. 2003. A cobertura florestal da Bacia do Rio Itajaí: elementos para uma análise histórica. Tese de doutorado, Universidade Federal de Santa Catarina, Florianópolis.

Windisch, P.G. 2002. Pteridófitas do Brasil: diversidade decrescente. In: E.L. Araujo, A.N. Moura, E.V.S.B. Sampaio, L.M.S. Gestinari \& J.M.T. Carneiro (eds.). Biodiversidade, conservação e uso sustentável da flora do Brasil. Universidaade Federal Rural de Pernambuco e Sociedade Botânica do Brasil, Recife, pp. 196-198.

Zuloaga, F.O., Morrone, O. \& Beltrano, M.J. 2008. Catálogo de las plantas vasculares del Cono Sur. Monographs in Systematic Botany from the Missouri Botanical Garden 107: 1-161. 